\title{
Examining the relationship between the NUTRIC 2002 score and the complications and mortality up to 48 hours after the discharge of multiple trauma patients admitted to the ICU of Alzahra Hospital in Isfahan (2018-2019)
}

\author{
BABAK ALI KIAII ${ }^{1}$, SEYED TAGHI HASHEMI ${ }^{2 *}$, SARA MOUSAVI ${ }^{3}$, NAFISEH SAFIAN ${ }^{4}$ \\ ${ }^{1}$ Associate Professor of Intensive Care, Medical School, Isfahan University of Medical Sciences, Isfahan, Iran- Email: \\ alikiaiib@med.mui.ac.ir \\ ${ }^{2}$ Assistant Professor, Anesthesiologist, and Intensive Care Unit Specialist, Medical School, Isfahan University of Medical Sciences, Isfahan, \\ Iran.-Email: st_hashemi@med.mui.ac.ir \\ ${ }^{3}$ Assistant Professor of Clinical Pharmacy, University of Isfahan - Email: s.mousavi@pharm.mui.ac.ir \\ ${ }^{4}$ Professional Medical Doctor, Medical School, Isfahan University of Medical Sciences, Isfahan, Iran - nafis.safian@gmail.com
}

\begin{abstract}
Introduction: Eating disorders are among the most common problems in patients suffering from multiple trauma admitted to the ICU. They have a considerable impact on the increase of mortality risk. The present study aimed to determine the relationship between the Nutrition Risk in Critically ill (NUTRIC) score and the complications and mortality up to 48 hours after the discharge of multiple trauma patients admitted to the ICU of Alzahra Hospital in Isfahan between 2018 and 2019.

Methodology: This study was descriptive-analytical research on 68 multiple trauma patients admitted to the ICU of Alzahra Hospital. The NUTRIC 2002 scores of the said patients were calculated by evaluating the parameters of age, APACHE II, SOFA Score, days in the hospital to ICU admission, and the number of comorbidities. Two different study groups were compared in terms of the said parameters, one composed of living and the other of deceased subjects.

Findings: The mean NUTRIC 2002 Score was $3.5 \pm 0.03$ in all studied patients. Out of the said patients, $67.6 \%$ and $32.4 \%$ of them were exposed to a low-risk eating disorder and a high-risk eating disorder, respectively. The mean of NUTRIC Scores in living and deceased patients were $2.82 \pm 3$ and $1.19 \pm 5.83$, respectively; which indicated that the deceased subjects had a higher NUTRIC Score $(p<0.001)$.

Conclusion: The 2002 NUTRIC Score was higher in the deceased subjects than in the living patients. Thus, this score seems to be a valuable and usable criterion for determining the eating disorder and the risk of mortality in multi-trauma patients admitted to the ICU.

Keywords: NUTRIC, Multiple Trauma, Intensive Care, Death
\end{abstract}

\section{INTRODUCTION}

In cases of trauma, the body's metabolic and systemic response to stress causes a considerable increase in the metabolism and catabolism of protein stores, which leads to tissue mass depletion, weight loss, and negative nitrogen balance. As a result, it accelerates the development and progression of malnutrition and its consequent infectious complications and increases the mortality rate $(5-1)$.

Malnutrition is one of the most serious and common problems in the ICU, which has adverse consequences such as immune system dysfunction, increased infections, delayed wound healing process, increased dependency on mechanical ventilation, prolonged recovery time, increased length of hospitalization, increased hospital costs, and enhanced mortality rate. Therefore, preventing or minimizing the risk of malnutrition is essential to improve the clinical complications of patients admitted to the ICU (6 and 7).

The Severity of Illness Scoring Systems is important tools for assessing disease prognosis. The primary effect in trauma patients is to assess the risk of mortality. As the incidence of trauma increased, many statistical models were developed with the aim of objective prognosis of trauma patients. Such systems, like APACHE II, Saps, and NUTRIC 2002 are now widely used for critically ill patients. Besides, the SOFA scoring system has recently been developed and validated. Trauma scoring systems have been developed to start patients' triage in various areas. Such systems require an easy and ergonomic user guide (8).

Adequate nutrition in trauma patients admitted to the ICU is crucial due to the increased metabolism, calorie, and protein requirements. It is also closely related to the mortality and morbidity of patients (9).

The NUTRIC criterion is a tool used for assessing the nutritional status of patients admitted to the ICU. However, despite its practicality and importance, so far no study has focused on evaluating this criterion in multiple trauma patients. Hence, given that eating disorders are one of the leading causes of death in the ICU and there is still no precise system in the ICU that measures the mortality and morbidity of patients based on the existing standards, the present study has aimed to examine the NUTRIC 2002 score for multiple trauma patients as a predictor of mortality and morbidity.

\section{MATERIALS AND METHODOLOGY}

This research was a cross-sectional study between 2018 and 2019 in the ICU of Alzahra Hospital in Isfahan. The statistical population of this study consisted of multiple trauma patients admitted to the said unit.

For the patients to partake in the study, they had to be $18+$ years old, suffer from multiple trauma, and agree to 
share their personal information. Moreover, patients suffering from severe malnutrition, psychiatric disorders leading to severe malnutrition, patients with coma whose feeding history was unknown, patients treated with appetite suppressants, and pregnant and lactating patients were not included in the study.

Death before 24 hours of hospitalization in the ICU and resistant vomiting (resistant to metoclopramide treatment) were considered exclusion criteria.

The sample size was estimated using the formula for correlation studies with a $95 \%$ confidence level, $80 \%$ test power, the correlation between the SOFA index and NUTRIC 2002 score (0.329) (28). Using the said criteria, the sample size was estimated to be 70 patients. The convenience non-probability sampling technique was used to select the research sample and all patients who were eligible to participate in the research were included in the study based on the time of their admission to the ICU until 70 patients were selected and the sample size reached the desired size.

The selected 70 patients entered the study after obtaining permission from the Medical Ethics Committee of Isfahan University. The NUTRIC score was calculated at the time of admission for multiple trauma patients admitted to the ICU as an emergency. The NUTRIC 2002 scores were calculated by evaluating the parameters of age, APACHE II, SOFA Score, days in the hospital to ICU admission, and the number of comorbidities (between 0 and 9).

A score of 5-9 was a high score indicating high mortality and eating disorders, and lower scores showed low eating disorders. Also, APACHE II and SOFA scores are used in this scoring system, and these measures were also examined in the ICU.

Numbers of comorbidities include high blood pressure, diabetes, chronic kidney disease, neurological diseases, coronary artery disease, chronic airway obstruction, liver disease, and malignancy.

After calculating the NUTRIC score at the beginning of ICU admission, mortality and morbidity rates up to 48 hours after discharge from ICU were examined. These factors were assessed and recorded by a physician during daily visits. The aforementioned complications included pneumonia, heart problems, psychological complications, kidney complications, and stroke.

Pneumonia was assessed using the SIRS criterion including body temperature above 38 or below $36^{\circ} \mathrm{C}$, tachypnea (respiratory rate of fewer than 24 beats per minute), tachycardia (heart rate of greater than 90 beats per minute), leukocytosis (more than 12000 units per liter) or leukopenia (less than 4000 units per liter) of more than $10 \%$.

Heart problems included cardiac arrhythmia or atrial fibrillation due to electrocardiography and myocardial infarction, given the ECG and increased troponin.

Psychological complications were evaluated based on the CAM-ICU screening tool which has 4 items: acute change in one's mental state (1), lack of attention (2), thinking disorder (3), and changes in their awareness level (4). Using this method, delirium was positive if criterion 1 was associated with criterion 2 and one of the criteria was 3 or 4 that this tool had yes and no answers.

Renal complications included acute renal failure in the form of urea levels less than $0.5 \mathrm{ccs} / \mathrm{kg} / \mathrm{h}$. Stroke was determined based on clinical symptoms, decreased level of consciousness, and CT scan findings.

Finally, the obtained data were entered into SPSS software version 22 and analyzed using the statistical tests of Chi-square and T-test and logistic regression test.

\section{RESULTS}

In this research, 70 patients suffering from multiple trauma admitted to the ICU of Alzahra Hospital were studied. Out of these 70, 2 patients were excluded from the study due to death before 24 hours. The age range of patients was between 19 and 76 years (mean $=38.6 \pm 7.16$ ). In the research sample, 48 patients $(70.6 \%)$ were under 50 years old and 20 patients (29.4\%) were $50+$ years old. Furthermore, 45 patients $(66.2 \%)$ were male and 23 patients $(33.8 \%)$ were female. Figure 1 showed the age distribution of patients by gender.

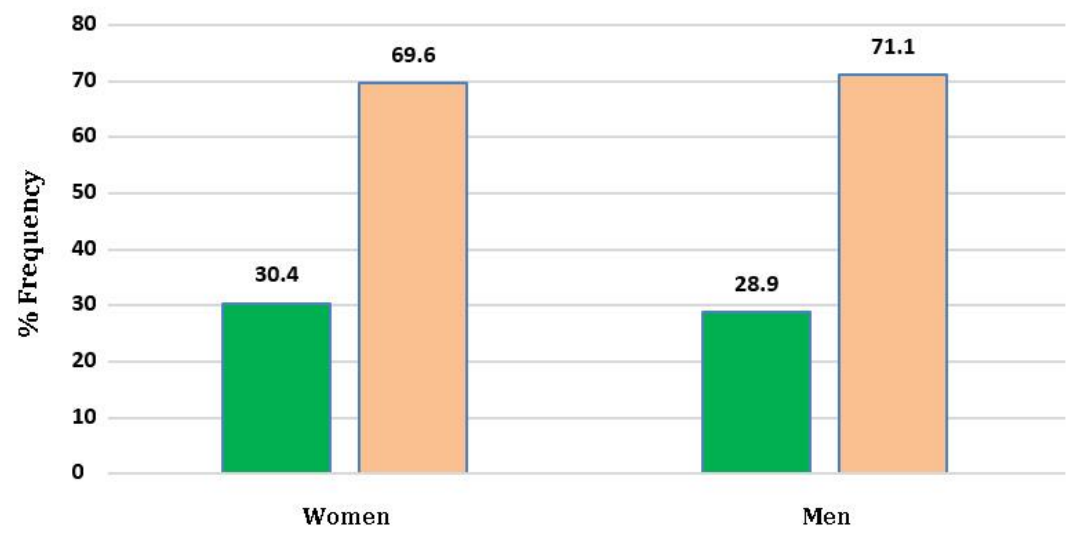

$\square$ Below 50 years old $\quad \square 0$ years old and above

Figure 1: Frequency percentage of age group based on gender 
The mean APACHE II score in the studied patients was $16.04 \pm 5.23$ with a range of $1-25$. According to the mentioned score, there were death risks of $10 \%$ (scores 0 15), $15 \%$ (scores 16-19), and $35 \%$ (scores 20-30) in 32, 17, and 19 patients, respectively.

The mean SOFA score was $3.88 \pm 7.88$ with a range of 2-23. Additionally, the mean days in the hospital to ICU admission was $1.82 \pm 0.73$ with a range of $1-3$ days. Out of the examined patients, 22 patients $(32.4 \%)$ suffered from comorbidities, the most common of which were hypertension and diabetes. Out of the research sample, 9 patients (13.2\%), $9(13.2 \%), 3(4.4 \%)$, and 1 patient $(1.5 \%)$ had $1,2,3$, and 4 concomitant diseases, respectively.
Table 1 showed the frequency distribution of the said indicators in all patients by age and gender. According to table 1, there was no difference between the mean scores of APACHE II, SOFA, days in the hospital to ICU admission, and the number of comorbidities based on gender. However, this was not the case for the mean scores of APACHE II, SOFA, days in the hospital to ICU admission, and the number of comorbidities based on age. There was a significant difference between patients in the age groups of below 50 and $50+$ years in terms of the said scores.

Table 1: Frequency distribution of vital signs in all patients by age and gender

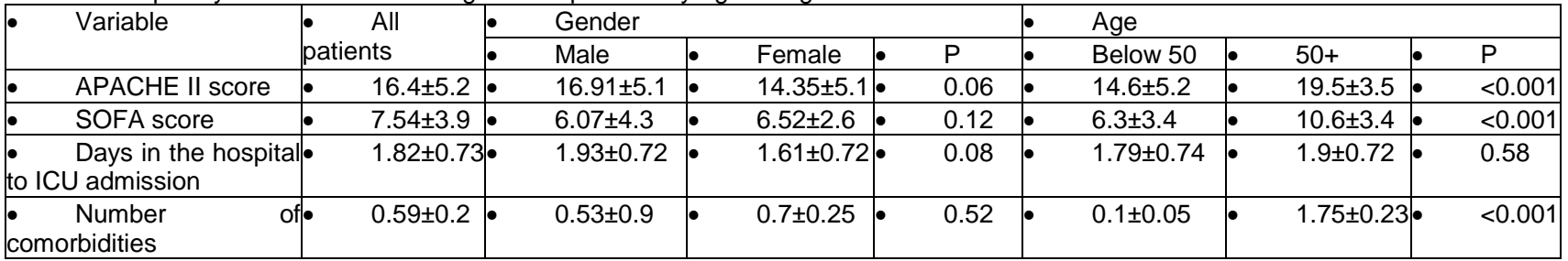

The mean of NUTRIC score 2002 in all patients was $3.5 \pm 0.03$ with a range of $1-8$. Out of the 68 studied patients $(67.6 \%)$ were exposed to low eating disorders and 22 patients $(32.4 \%)$ were exposed to high eating disorders.

The mean NUTRIC scores in men and women were $3.69 \pm 0.04$ and $3.13 \pm 2$, respectively, Thus, there was no significant difference between the two genders $(P=0.29)$. The mean NUTRIC scores in people under 50 and $50+$ years and older were $2.5 \pm 1.37$ and $5.9 \pm 1.17$, respectively; meaning that the aforementioned score was significantly higher in people who were 50 and older $(\mathrm{P}<0.001)$ (Figure 2).

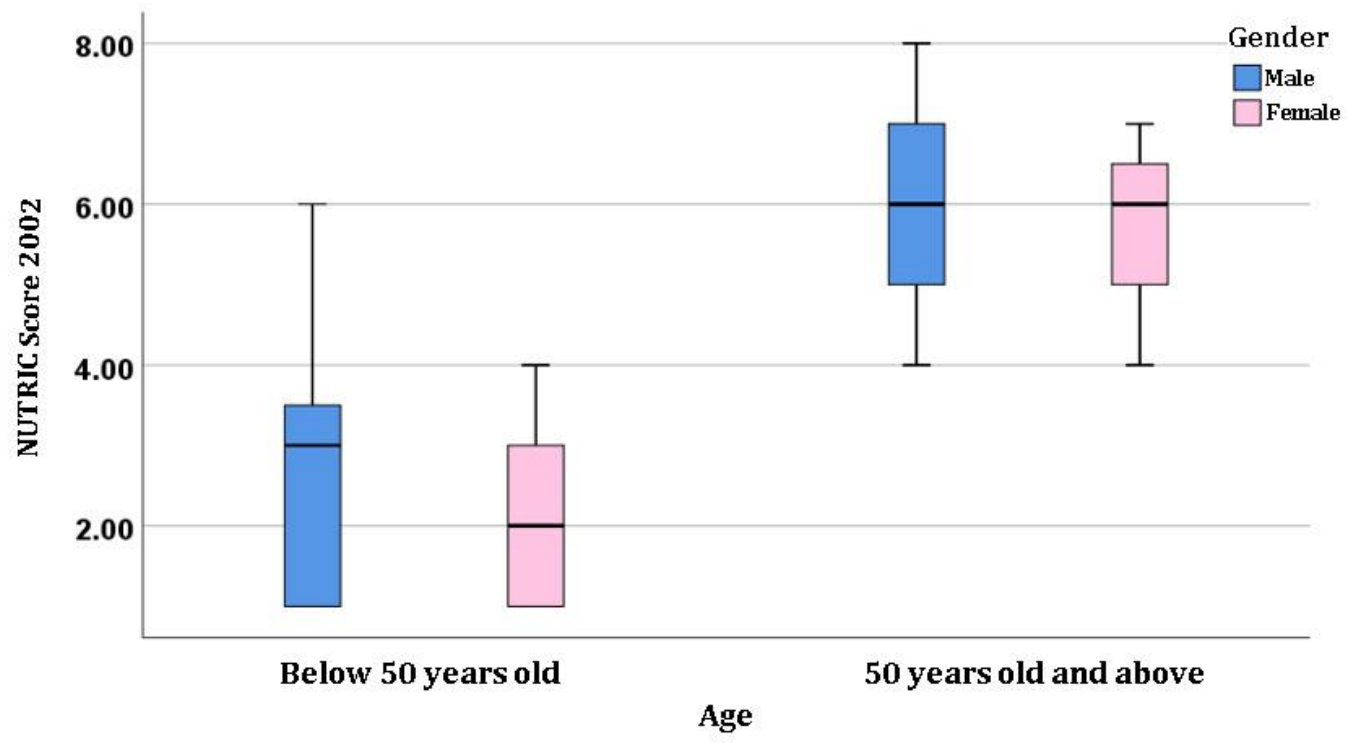

Figure 2: Mean, range, and percentile of $25-75 \%$ of the NUTRIC score by age and gender

Out of the 68 patients, $12(17.6 \%)$ died during their stay in the intensive care unit. The mean ages of the living and deceased patients were $36.45 \pm 15.9$ and $17.6 \pm 48.75$, respectively; indicating that the deceased patients had a higher mean age $(P=0.02)$. Besides, 35 of the living patients and 10 of the deceased patients were males $(62.5 \%$ vs. $83.3 \%)$, but there was no significant difference between the patients in terms of gender $(\mathrm{P}=0.17)$.
The mean NUTRIC scores of the living and the deceased patients were $3 \pm 2.82$ and $5.83 \pm 1.19$, respectively; indicating that the deceased patients had a higher NUTRIC score. The mean scores of APACHE II and SOFA were also significantly higher in the deceased patients $(P<0.001)$. On the other hand, the frequency of high nutritional risk in deceased patients was significantly higher. In other words, the frequencies of high nutritional 
risk in the living and the deceased patients were $21.4 \%$ and $83.3 \%$, and the difference between the living and deceased groups was significant $(p<0.001)$.

The difference between the living and the deceased groups was not significant $(P=0.36)$, despite the number of comorbidities being higher in the deceased group (Table 2 ). According to the logistic regression test, for each unit of increase in the NUTRIC score, the risk of mortality in patients increased by 2.44 times $(95 \%$ confidence level: 1.49-3.99), which was statistically significant $(\mathrm{P}<0.001)$. Other variables, did not have a significant impact on the risk of mortality, including age, sex, and complications of the disease.

Table 2: distribution of the demographic and clinical variables in the two groups of the living and the deceased patients

\begin{tabular}{|c|c|c|c|c|}
\hline \multirow{2}{*}{\multicolumn{2}{|c|}{ Variable }} & \multicolumn{2}{|l|}{ State } & \multirow[t]{2}{*}{$\mathrm{P}$} \\
\hline & & Living & Deceased & \\
\hline \multicolumn{2}{|l|}{ Mean age (year) } & $36.45 \pm 15.9$ & $48.75 \pm 17.6$ & 0.02 \\
\hline \multirow[t]{2}{*}{ Age group } & Below 50 years & $54(76.8)$ & $5(41.7)$ & \multirow[t]{2}{*}{0.015} \\
\hline & 50 years and older & $13(23.2)$ & $7(58.3)$ & \\
\hline \multirow[t]{2}{*}{ Gender } & Male & $35(62.5)$ & $10(83.3)$ & \multirow[t]{2}{*}{0.17} \\
\hline & Female & $21(37.5)$ & $2(16.7)$ & \\
\hline \multicolumn{2}{|c|}{ Mean APACHE II score } & $14.79 \pm 4.65$ & $21.92 \pm 3.55$ & \multirow{4}{*}{$\begin{array}{l}<0.001 \\
<0.001\end{array}$} \\
\hline Mortality risk based on & $10 \%$ & $31(55.4)$ & $1(8.3)$ & \\
\hline \multirow[t]{2}{*}{ the APACHE II score } & $15 \%$ & $16(28.6)$ & $1(8.3)$ & \\
\hline & $35 \%$ & $9(16.1)$ & $10(83.3)$ & \\
\hline \multicolumn{2}{|l|}{ Mean SOFA score } & $6.52 \pm 2.75$ & $12.33 \pm 4.87$ & $<0.001$ \\
\hline \multicolumn{2}{|l|}{ Mean NUTRIC score } & $3 \pm 1.82$ & $5.83 \pm 1.19$ & $<0.001$ \\
\hline \multirow[t]{2}{*}{ Risk of eating disorders } & Low & $44(78.6)$ & $2(16.7)$ & \multirow{2}{*}{$<0.001$} \\
\hline & High & $12(21.4)$ & $10(83.3)$ & \\
\hline \multicolumn{2}{|c|}{ Days in the hospital to ICU admission } & $1.79 \pm 0.73$ & $2 \pm 0.74$ & 0.36 \\
\hline \multicolumn{2}{|c|}{ Suffering from comorbidities } & $15(26.8)$ & $7(58.3)$ & 0.034 \\
\hline \multicolumn{2}{|c|}{ Number of comorbidities } & $0.46 \pm 0.11$ & $1.17 \pm 0.39$ & 0.023 \\
\hline \multicolumn{2}{|c|}{ Incidence of complications during hospitalization } & $24(42.9)$ & $12(100)$ & $<0.001$ \\
\hline
\end{tabular}

According to the study findings, out of the 68 studied patients, 37 patients (54.4\%) had complications during hospitalization. The frequencies of complications during hospitalization were 25 (44.6) and 12 (100\%) in the deceased and the living patients, respectively. The frequency of complications during hospitalization was significantly higher in the deceased $(P<0.001)$. The most common complication in the studied patients was pneumonia with a frequency of $38.2 \%$. Other complications included infection (27.9\%), psychological complications (19.1\%), renal complications (17.6\%), cardiac complications $(2.13 \%)$ and cerebral complications such as stroke $(9.7 \%)$. It must be noted that out of the 37 patients with complications during hospitalization, only 11 (29.7\%) had one complication during their hospitalization and the other $26(70.3 \%)$ had more than one complication during their hospitalization.

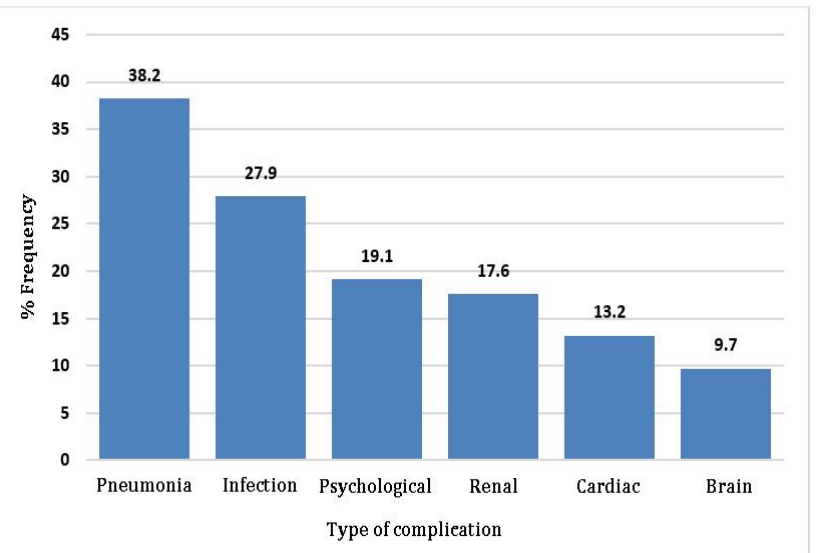

\section{DISCUSSION}

Predicting the mortality risk of multiple trauma patients admitted to the ICU is one of the most important measures to take for determining how the patient is medically cared for. In this respect, numerous criteria and indicators have been presented, including APACHE and SOFA scores. On the other hand, nutritional problems are among the most serious problems of patients admitted to ICU. Such problems can have a considerable impact on increasing the risk of mortality (10). However, despite the importance of this issue, it has yet to be studied thoroughly. In this regard, the NUTRIC 2002 score is one of the criteria used for evaluating the nutritional status of patients admitted to the ICU. Therefore, the present study aimed to determine the relationship between the NUTRIC 2002 score and the complications and mortality up to 48 hours after the discharge of multiple trauma patients admitted to the ICU of Alzahra Hospital in Isfahan.

Examining 68 multiple trauma patients admitted to the ICU showed that more than $70 \%$ of these patients were younger than 50, and there were twice as many men as women. Since multiple trauma is mainly caused by traffic accidents or occupational incidents, young and active people, especially men, are at risk of this type of trauma. Besides, due to the severity of internal organ damages, nutritional problems and eating disorders are more common among patients admitted to the ICU (11).

Based on the findings of our study regarding the NUTRIC score, $32.4 \%$ of the patients suffering from multiple trauma were at risk of high-risk eating disorders. In the study by Kalaiselvan et al., 678 patients admitted to the ICU under mechanical ventilation were examined. Out of these patients, $42.5 \%$ were at risk of severe eating disorders (12). These findings were compatible with the 
findings of our study. Kondrup et al. also reviewed five studies on the evaluation of the nutritional status of critically ill patients admitted to ICUs. According to their findings, four studies confirmed the positive effect of the NUTRIC score in estimating the nutritional status of the patients and their recovery. In their study, it was shown that the NUTRIC measure was a valuable tool for assessing the nutritional status of patients admitted to the ICU; while the other scores, such as APACHE, could not explain the patients' nutritional status (13).

According to the findings of the present study, the mean NUTRIC score in the deceased patients was significantly higher than the living patients. On the other hand, the frequency of high-risk eating disorders in the deceased patients was significantly higher than the frequency of this variable in the living patients. Moreover, in this study, for each unit of increase in the NUTRIC score, the risk of mortality in patients increased by 2.44 times. In the study by Rahman et al., 1199 patients in the ICU were examined in terms of their nutritional status. According to their findings, for each unit of increase in the NUTRIC score, the risk of mortality in patients increased by 1.4 times (14). In another study by Mukhopadhyay et al., 40 critically ill patients admitted to the ICU were examined. They concluded that for each unit of increase in the NUTRIC score, the risk of mortality in patients increased by 1.48 times 28 days after ICU admission (15).

Based on the findings of the present study, there were significant differences between the two groups (the living and the deceased) in terms of the studied parameters, i.e. age, APACHE II, SOFA Score, days in the hospital to ICU admission, and the number of comorbidities. The findings also showed that the frequency and mean of the said parameters were higher in the deceased patients.

\section{CONCLUSION}

The NUTRIC 2002 score was higher in the deceased patients than the living ones; thus, this score seems to be a valuable and beneficial criterion for determining the eating disorders and the risk of mortality in multiple trauma patients admitted to the ICU. Nonetheless, due to the limitations of the present study, such as the small sample size and short follow-up period, it is suggested to focus more on this subject and conduct more studies in this field.

\section{REFERENCES}

1. Woien H,Torunn Bjork, Nutrition of the critically ill patient and effects of implementing a nutritional support algorithm in ICU. Journal of Clinical Nursing, 2006. 15: p. 168-177
2. Mahan L.K, Escott-Stump S, Krause's Food \& Nutrition Therapy.twelve edition ,SAUNDERS Elsevier,Philadelphia, 2008: p. 383.

3. Carayon P, Gurses A P. Patient Safety and Quality: An Evidence-Based Handbook for Nurses, chapter 30. p.1-14.

4. Chen Y-C,Chou Sh-Sh,Lin L-H,Wu L-F, The Effect of Intermittent Nasogastric Feeding on Preventing Aspiration Pneumonia in Ventilated Critically III Patients. Journal of Nursing Research, 2006. 14(3): p. 167-179.

5. MacLeod JB, Lefton J, Houghton D,Roland C, Doherty J, Cohn S.M, Barquist E.S, Prospective Randomized control Trial of Intermittent Versus Continuous Gastric feeds for Critically III Trauma Patients. J Trauma, 2007. 63(1): p. 5761.

6. Smeltzer S C, Bare B G,Hinkle J L, Cheever K H, Brunner \& Suddarth`s Textbook of Medical-Surgical Nursing, eleven edition, Lippincott Williams \& Wilkins,Philadelphia,2008: p. 1182-1185, 1200.

7. M.N. Chawdaa, F. Hildebrandb, H.C. Papeb, P.V. Giannoudis, Predicting outcome after multiple trauma: which scoring system?, Injury, Int. J. Care Injured, 2004. 35: p. 347-358

8. Hwang SY, Lee JH, Lee YH, Hong CK, Sung AJ, Choi YC. Comparison of the Sequential Organ Failure Assessment, Acute Physiology and Chronic Health Evaluation II scoring system, and Trauma and Injury Severity Score method for predicting the outcomes of intensive care unit trauma patients. The American journal of emergency medicine. 2012;30(5):749-53.

9. Marik $P$ E, Zaloga G P, Early enteral nutrition in acutely ill patients:A systematic review. Critical Care Medicine, 2001. 29(12): p. 2264-2270.

10. Minard G,Kudsk K A,Melton Sh,Patton J H,Tolley E A, Early Versus Delayed Feeding With an Immune-Enhancing Diet in Patient with Severe Head Injuries. JPEN, 2000. 24(3): p. 145-149.

11. Wick M, Ekkernkamp A, Muhr G. The epidemiology of multiple trauma. Der Chirurg; Zeitschrift fur alle Gebiete der operativen Medizen. 1997 Nov;68(11):1053-8.

12. Kalaiselvan MS, Renuka MK, Arunkumar AS. Use of nutrition risk in critically ill (nutric) score to assess nutritional risk in mechanically ventilated patients: A prospective observational study. Indian journal of critical care medicine: peer-reviewed, official publication of Indian Society of Critical Care Medicine. 2017 May;21(5):253.

13. Kondrup J. Nutritional-risk scoring systems in the intensive care unit. Current Opinion in Clinical Nutrition \& Metabolic Care. 2014 Mar 1;17(2):177-82.

14. Rahman A, Hasan RM, Agarwala R, Martin C, Day AG, Heyland DK. Identifying critically-ill patients who will benefit most from nutritional therapy: further validation of the "modified NUTRIC" nutritional risk assessment tool. Clinical nutrition. 2016 Feb 1;35(1):158-62.

15. Mukhopadhyay A, Henry J, Ong V, Leong CS, Teh AL, van Dam RM, Kowitlawakul Y. Association of modified NUTRIC score with 28-day mortality in critically ill patients. Clinical nutrition. 2017 Aug 1;36(4):1143-8. 